durch eine okuläre Myasthenie überlagert wurde. Weiters finden sich Zeichen einer milden bis mäßigen Generalisierung im Bereich der Extremitäten (IIa). Die Diagnose konnte durch die typische Klinik gestellt werden, die im Rahmen der Durchuntersuchung erhobenen Befunde waren zur Gänze negativ. Eine Therapie mit Acetylcholinesterase-Hemmern und Glukokortikoiden wurden vom Patienten schlecht vertragen. Die derzeitige Therapie mit Immunglobulinen bessert den Leistungsabbau im Bereich der Extremitäten, nicht jedoch die okuläre Symptomatik.

Fall 2 (weiblich, geb. 1967) beschreibt eine okuläre Myasthenie mit einer Generalisierung unter Beteiligung der oropharyngealen Muskulatur (IIb). Im Unterschied zu Fall 1 fand sich eine Abnormität des Thymus. Die Therapie mit Mestinon und Glukokortikoiden besserte die okuläre Symptomatik. Nach Thymektomie konnte die Therapie jedoch nur vorübergehend ausgesetzt werden. Besonders die Gefahr einer Insuffizienz der Schlund- und Atemmuskulatur mit der drohenden Erstickungsgefahr rechtfertigt die derzeitige Therapie mit Immunglobulinen.

Gemeinsam ist beiden Fällen eine vorwiegend okuläre Symptomatik mit einer eher mäßigen Beeinflussbarkeit der okulären Myasthenie durch die medikamentöse Therapie. Die derzeitige Immunglobulintherapie bessert in erster Linie die Zeichen einer Generalisierung der Erkrankung. Eine Immunsuppression durch Azathioprin wurde bisher durch Patient 1 wegen Gefahr der Myelotoxizität, durch Patient 2 wegen einer möglichen Gravidität abgelehnt.

\title{
Okuläre Myasthenie oder was sonst? - Fallbericht
}

M. Koch und A. Langmann

Graz

Zusammenfassung. Die Autoimmunerkrankung Systemischer Lupus Erythematodes vermag aufgrund rein klinisch imponierender Augenmuskelstörungen und der Ausbildung eines positiven Acetylcholinrezeptor-Ak-Titers eine Myasthenia gravis zu imitieren. Die Mitbeteiligung der Lidmuskulatur und der extraokularen Muskulatur kann als Ausdruck der zerebralen Manifestation oder aber als Folge der Autoantikörperbildung gegen die Acetylcholinrezeptoren gesehen werden. Letzteres erscheint aufgrund der wechselnden Symptomatik und der stark schwankenden Motilitätsverhältnisse wahrscheinlicher. Die Papillenschwellung beidseits wurde bei dieser Patientin aufgrund der zusätzlich vorhandenen Randblutungen und des Cotton-wool-Herdes auf eine vaskuläre Ursache zurückgeführt. Eine Neuritis bzw. Papillitis kam differentialdiagnostisch nicht in Betracht, da in diesem Fall eine Störung des Kontrastsehens, ein reduzierter Visus oder ein pathologisches VEP zu erwarten gewesen wären.

Der Systemische Lupus Erythematodes, das Chamäleon der Medizin, vermag viele Krankheiten vorzutäuschen und unterstreicht daher die Wichtigkeit der Teamarbeit zwischen Orthoptisten, Ophthalmologen und anderen Disziplinen.

Schlüsselwörter: Wechselnde Augenmuskelparesen, Papillenschwellung, Lupus Erythematodes, Myasthenia gravis.

\section{Einleitung}

Der Systemische Lupus Erythematodes ist eine schubweise verlaufende chronisch-entzündliche Autoimmunerkrankung, welche Haut, Gelenke und innere Organe befallen kann. Okuläre Manifestationen treten vor allem bei Patienten während eines akuten Schubs der Erkrankung auf und können aufgrund rein klinischer Symptome und der Ausbildung von Acetylcholinrezeptor-Antikörpern den Verdacht auf die Autoimmunerkrankung Myasthenia gravis lenken.

\section{Fallbeschreibung}

Anamnese: Ein 11-jähriges Mädchen stellte sich aufgrund intermittierender Cephalgien, einer vorübergehenden Ptosis rechts sowie horizontaler Doppelbilder an unserer Klinik vor.

Ophthalmologischer und orthoptischer Befund: Bei der Vorstellung in unserer Ambulanz präsentierte sich eine geringe Abduzensparese links und eine nunmehr linksseitige Ptosis. Aufgrund dieser rein klinischen Symptome wurde die Verdachtsdiagnose einer Myasthenia gravis gestellt und durch den positiven Acetylcholinrezeptor-Ak-Titer bestätigt. Im Rahmen der Fundusuntersuchung zeigte sich jedoch beidseits eine ödematöse, 1-2 dpt. prominente Papillenschwellung mit Randblutung, welche mit einer Myasthenie nicht in Zusammenhang gebracht werden konnte. Weitere abklärende Untersuchungen, wie der Fernvisus, das Gesichtsfeld, die musterevozierten Potentiale und das Farbsehen waren unauffällig. Zum Zeitpunkt der zweiten orthoptischen Untersuchung konnte die Abduzensparese nicht mehr nachgewiesen werden, es hatte sich jedoch nun eine inkomplette äußere Oculomotoriusparese beidseitig ausgebildet.

Serologie: Nachdem in der serologischen Untersuchung sowohl ein pathologischer Cytomegalie-Ak-Titer als auch ein pathologischer Borrelien-Ak-Titer festgestellt worden waren, wurden weitere abklärende immunologische Untersuchungen veranlasst. Der deutlich erhöhte Anti-DNA-AntikörperTiter und die pathologischen Leber- und Nierenwerte führten schließlich zur Diagnose des Systemischen Lupus Erythematodes.

Therapie: Die Therapie der Wahl bestand in der Verabreichung einer Kortisonstoßtherapie, welche aufgrund der pathologischen Nieren- und Leberwerte mit einem Immunsuppressivum ergänzt werden musste. Unter dieser Therapie kam es zu einer vollständigen Rückbildung der Papillenschwellung und der Motilitätsstörungen. 